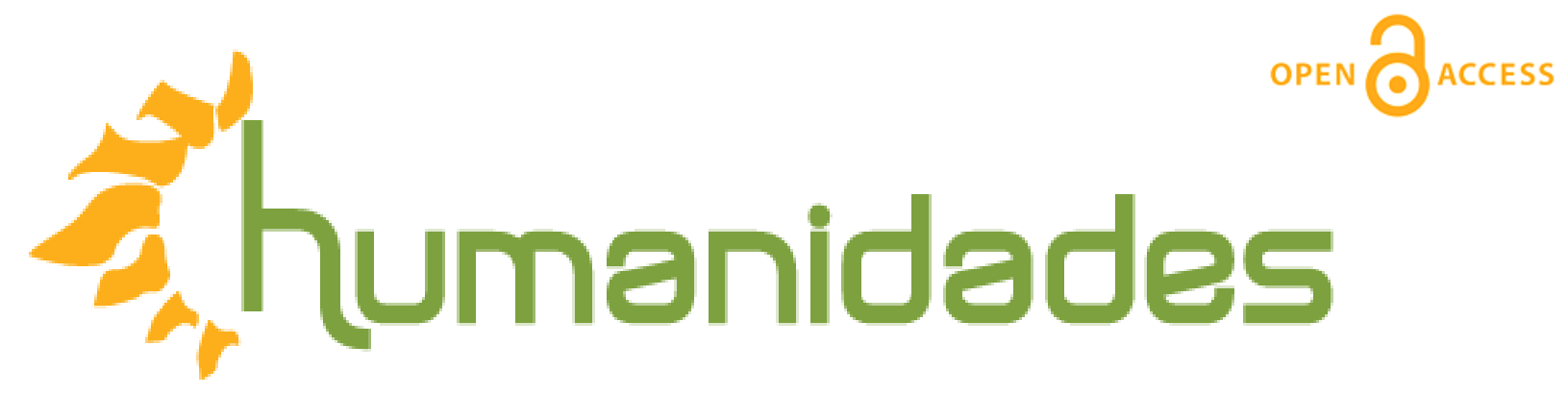

Revista de la Escuela de Estudios Generales, Universidad de Costa Rica

Enero-junio, 2017 • Volumen 7, número 1 • EISSN 2215-3934• pp. 1-37

Recibido: 14-Julio-2016 Aceptado: 02-Octubre-2016

\title{
Cuando los límites importan: Dimensión pública e implicancias sociales del trabajo arqueológico
}

DOI: http://dx.doi.org/10.15517/h.v7i1.27640

Virginia Salerno, Milva Umaño, Verónica Helfer, Alejandra Raies, H. César Pinochet, Mariano Darigo, Matías Warr y Mariano Ramos

Todos los derechos reservados. Universidad de Costa Rica. Esta revista se encuentra licenciada con Creative Commons. Reconocimiento-NoComercial-SinObraDerivada 3.0 Costa Rica.

Correo electrónico: humanidades@ucr.ac.cr / Sitio web: http://revistas.ucr.ac.cr/index.php/humanidades 
Virginia Salerno: Instituto de Arqueología, Facultad de Filosofía y Letras, Universidad de Buenos Aires, Argentina. Consejo Nacional de Investigaciones Científicas y Técnicas (CONICET). Correo: vmasalerno@gmail.com

Milva Umaño: Programa de Arqueología Histórica y Estudios Pluridisciplinarios (PROARHEP) y Universidad Nacional de Luján, Argentina.Correo:milvauma@ hotmail.com

Verónica Helfer: Programa de Arqueología Histórica y Estudios Pluridisciplinarios (PROARHEP) y Universidad Nacional de Luján, Argentina. Correo: veronicahelfer@hotmail.com

Alejandra Raies: Programa de Arqueología Histórica y Estudios Pluridisciplinarios (PROARHEP) y Universidad Nacional de Luján, Argentina. Correo: alejandraraies@gmail.com

H. César Pinochet: Programa de Arqueología Histórica y Estudios Pluridisciplinarios (PROARHEP) y Universidad Nacional de Luján, Argentina. Correo: hcpinochet@ gmail.com

Mariano Darigo: Programa de Arqueología Histórica y Estudios Pluridisciplinarios (PROARHEP) y Universidad Nacional de Luján, Argentina. Correo: ventolita@ hotmail.com

Matías Warr: Programa de Arqueología Histórica y Estudios Pluridisciplinarios (PROARHEP) y Universidad Nacional de Luján, Argentina. Correo: ventolita@ hotmail.com

Mariano Ramos: Programa de Arqueología Histórica y Estudios Pluridisciplinarios (PROARHEP) y Universidad Nacional de Luján, Argentina. Centro de Investigaciones en Antropología Filosófica y Cultural (CIAFIC-CONICET). Correo: onairamsomar@ gmail.com 


\title{
Cuando los límites importan: Dimensión pública e implicancias sociales del trabajo arqueológico
}

\section{Resumen}

Este trabajo aporta una mirada reflexiva sobre el impacto de una investigación arqueológica en el contexto local donde esta se desarrolla. Se presenta el caso del proyecto de investigación en el paraje El Tonelero de la localidad de Ramallo (Provincia de Buenos Aires, Argentina), donde se iniciaron trabajos de Arqueología histórica en el año 2012 como parte de un proyecto que estudia la guerra del Paraná, desde una perspectiva pluridisciplinar. Se discuten las implicancias de la construcción de una "dimensión pública" en torno a dicha investigación, con el propósito de indagar el tipo de relaciones que confluyen con los contenidos históricos investigados.

Palabras claves:

Arqueología, conflicto social, historia latinoamericana, espacio urbano, institucionalización.

\section{When Limits that Matter: Public Dimension and Social Impacts on Archaeological Practice}

\begin{abstract}
This paper seeks to provide a thoughtful look into the impact of archaeological research in the local context where it develops. With this aim we present the case of El Tonelero (Ramallo, Buenos Aires, Argentina), where we initiated an Historical Archaeological investigation began in 2012 as part of a projectwhich studies the Parana War -1845 and 1846- from a multidisciplinary perspective. We discuss the implications of the construction of a "public dimension" around this investigation, in order to analyze the type of relations that come together with historical contents investigated since the archaeology team started working at the place.
\end{abstract}

Keywords: Archaeology, social conflicts, Latin American history, urban spaces, institutionalization. 
El trabajo de investigación arqueológica se enmarca en una serie de Introducción relaciones institucionales y personales que se despliegan en una "dimensión pública" -en términos de visibilidad e interacciónmotorizando procesos de conocimiento y reconocimiento de actividades, sujetos, saberes y materiales asociados con el pasado en estudio; que muchas veces se encuentran en contradicción, por lo que generan tensiones en torno a la práctica arqueológica. Al respecto nos preguntamos: ¿en qué medida estas relaciones posibilitan que la investigación adquiera sentidos específicos en el contexto local?, ¿es posible que estas relaciones propicien procesos relacionados con la memoria del pasado que se busca investigar?

Con este fin presentamos el caso de un proyecto de investigación en el sitio El Tonelero de la localidad de Ramallo (Provincia de Buenos Aires, Argentina), donde iniciamos trabajos de Arqueología histórica en el año 2012 como parte de un proyecto que estudia la guerra del Paraná -1845 y 1846- desde una perspectiva pluridisciplinar. Discutimos las implicancias de la construcción de una dimensión pública en torno a dicha investigación, con el propósito de indagar el tipo de relaciones que confluyen con los contenidos históricos investigados desde que el equipo de arqueología se hizo presente en el lugar.

Se entiende la dimensión pública como una categoría de clasificación y organización social que delimita contextos diferenciados vinculados con formas de participación social y política, a la vez que permite establecer 
puntos de referencia socio-territoriales (Iazzeta, 2008; Ortiz, 2002; Moore, 1984). Entendida de esta manera, la dimensión pública de una investigación arqueológica se organiza a partir de las relaciones sociales que se construyen en forma situada, relacional y asimétrica cuando un equipo de investigación comienza a trabajar en un determinado lugar. En el marco de estas relaciones, diferentes aspectos materiales y simbólicos asociados con el pasado que se busca investigar pasan a formar parte del ámbito público. Sobre ello, se ha observado que cuando el pasado es parte del ámbito público se manifiesta la dimensión política de la memoria, en tanto la discusión/visibilidad pública sobre cómo un acontecimiento histórico hace posible la apelación a diferentes formas de memoria que conllevarían diferentes actuaciones en el presente (Rabotnikof, 1996).

De esto se desprende que la dimensión política es inherente a la investigación arqueológica, puesto que su desarrollo implica movilizar acciones, visualizar grupos de actores, destacar materiales y paisajes que en conjunto posibilitan elaborar criterios de valor sobre un pasado en un determinado lugar (Salerno y González, 2014). A partir de estas consideraciones entendemos que en la dimensión pública de una investigación arqueológica se tornan visibles las diferencias que en ocasiones articulan confrontaciones de distintas memorias, lenguajes y pasados. Por estos motivos, su estudio constituye un camino para indagar el modo en que una investigación arqueológica adquiere sentido en el contexto local. 
La investigación en el sitio El Tonelero tiene sus antecedentes en el pueblo vecino, Vuelta de Obligado, sitio arqueológico que comenzamos a estudiar en el año 2000 dentro del mismo proyecto general. Allí se aborda un proceso histórico que se inició en 1838 y culminó con la llamada gdel Paraná en 1846. Esta guerra, desarrollada sobre la base de causas económico-políticas, enfrentó a la Confederación Argentina con las potencias europeas de Francia e Inglaterra. El conflicto bélico incluyó el bloqueo del Río de la Plata, tuvo algunas escaramuzas y cuatro batallas principales. Dos de estas tuvieron lugar en el actual territorio de la Provincia de Buenos Aires (Vuelta de Obligado y El Tonelero) y dos más en la Provincia de Santa Fe (Quebracho y San Lorenzo).

El proyecto de investigación arqueológica desarrolló una propuesta para el estudio de los campos de batallas que se dieron en el marco de esa guerra. En principio el proyecto se focalizó en la batalla de Vuelta de Obligado y luego se amplió incluyendo los otros eventos. Esta propuesta de investigación se enmarca dentro de la Arqueología del Conflicto y en particular, de la violencia, y se desenvuelve con una perspectiva pluridisciplinar que combina aportes de la Arqueología, la Historia, la Psicología, estudios del ambiente y otros campos del conocimiento (Ramos et ál., 2014).

\section{Arqueología histórica en el sitio El Tonelero}


Para este trabajo es relevante mencionar que tanto Vuelta de Obligado como El Tonelero son poblados satélites de ciudades mayores (San Pedro y Ramallo). Se trata de pequeños parajes instalados en las márgenes del río Paraná, con una población que no supera los 200 habitantes y que en gran parte están instalados en terrenos de los que no son o no eran propietarios y, a su vez, muchos de ellos no viven de forma permanente en el lugar, utilizando sus casas como lugares para pasar en fin de semana como en el caso de El Tonelero. Estos aspectos son cruciales a la hora de considerar el contexto en el que se desenvuelve la investigación arqueológica, pues permiten construir un marco de inteligibilidad sobre la manera en que los habitantes de estos parajes se relacionan con las múltiples narrativas de la historia del lugar.

Así por ejemplo, se ha observado que en el caso de Vuelta de Obligado la investigación arqueológica aporta elementos simbólicos de identificación que, en ocasiones, permitieron a los vecinos de Vuelta de Obligado diferenciarse socio-políticamente de los espacios territoriales mayores que integran (Salerno, 2014; Astorga, 2007).

En el caso de El Tonelero, desde que hicimos público el objetivo de estudiar el campo de batalla, habitantes del paraje y múltiples actores relacionados con él, plantearon sus expectativas e intereses en relación con la investigación arqueológica. Estos se asocian con la preservación del área como lugar histórico, potenciando su valor turístico y residencial, así como 
con la transformación del ambiente natural mediante la instalación de empresas y un puerto multirubros que pondrían el área en riesgo.

Si bien se trata de actores con diferentes intereses económicos y políticos, todos coinciden en que es necesario que el equipo de investigación establezca límites precisos en el sitio arqueológico investigado. La demanda de estos múltiples actores por establecer límites se relaciona con el supuesto de que el identificar una porción del territorio en términos de patrimonio arqueológico e histórico, conlleva su inclusión en un marco normativo proteccionista que limita sus potenciales usos y por estos motivos, sería un factor suficiente para dirimir una gran parte de los conflictos que se están desarrollando actualmente en el área.

En esta discusión, las posturas extremas se definen entre un grupo de actores que claman por definir todo el espacio de la costa ribereña como sitio patrimonial y reserva natural -lo que limitaría una gran parte de los proyectos económicos-; mientras que otro grupo reclama establecer una porción del territorio específico en el que se pudiera generar un espacio conmemorativo y liberar los potenciales usos del resto de la costa ribereña.

La guerra del Paraná se desarrolló en el marco del período de los Gobiernos de Juan Manuel de Rosas durante parte de la primera mitad del siglo XIX, desde 1829 a 1852 . Este personaje de la historia argentina ha sido una figura históricamente controvertida y su memoria fue parte de los temas tabú dentro la historia nacional de carácter oficial liberal (Ramos, 2009).

\section{La guerra del \\ Paraná y su \\ memoria en Vuelta \\ de Obligado y El \\ Tonelero}


En la historiografía argentina, la discusión sobre este tema se desarrolló a lo largo de casi todo el siglo XX. La figura de Rosas fue reivindicada por las corrientes del revisionismo histórico de las décadas de 1930, 1970 y la actual. Además, desde 1955 esta figura fue retomada como uno de los hitos políticos que sustentó una forma de nacionalismo a partir del tándem que formaban San Martín, Rosas y Perón.

Aunado a lo anterior, la figura de Rosas fue objeto de representación y resignificación en distintos ámbitos sociales y a través de diversos registros: canciones populares, poemas, cuentos, obras de arte y representaciones cinematográficas (Jacubowicz, 2005; Cattaruzza, 2007; Devoto y Pagano, 2009; Ramos, 2009).

En estos movimientos y sobre la base de esas perspectivas históricopolíticas, la batalla de Vuelta de Obligado se erigió como un hito significativo del Gobierno de la Confederación Argentina y en este marco, el campo de batalla fue uno de los anclajes para la producción y revisión de esta parte del pasado argentino. En consecuencia, el paraje Vuelta de Obligado fue escenario de diversas prácticas de memoria de la batalla que implicaron el movimiento de recursos económicos y simbólicos provenientes de distintas organizaciones políticas de la sociedad civil, así como del Estado nacional y provincial.

Durante la década de 1930, el revisionismo histórico se consolidó como nuevo actor historiográfico. Si bien esta línea de reflexión intelectual tiene 
sus antecedentes en los debates en torno al pasado argentino formulados en el marco del primer centenario de la Revolución de Mayo, sus aportes continuaron a lo largo de todo el siglo XX (Devoto y Pagano, 2009). En este marco se dio una producción de trabajos y obras generales que rebatieron las versiones de la Historia oficial. Entre los personajes de la historia nacional reivindicados, la figura de Juan Manuel de Rosas tuvo un lugar central (Devoto y Pagano, 2009; Cattaruzza, 2007).

Para esos años en Vuelta de Obligado se delimitó un parque conmemorativo señalando el área del campo de batalla, donde se inauguró un primer monumento junto con una cruz de ñandubay en homenaje a los caídos en la batalla. Además, el homenaje se reafirmó con la organización de un desfile militar, el oficio de una misa y un baile popular cada 20 de noviembre, fecha en que ocurrió el evento bélico (Ermaccora y Revello, 2009). Por su parte, el Estado Nacional en 1942 le otorgó al paraje el primer reconocimiento de carácter oficial, declarándolo "Lugar Histórico Nacional" mediante la Ley 120.411.

Desde entonces en el período que se extiende desde 1953 hasta 2015 se llevaron a cabo en Vuelta de Obligado diversidad de actos, oficiales y no oficiales, con la participación de actores locales y no locales que conllevaron distintas puestas en escena, con el movimiento de recursos económicos y simbólicos en pos de intervenir en la memoria de la batalla. Entre ellos pueden mencionarse autoridades militares $\mathrm{y}$ 
gubernamentales, agrupaciones políticas, movimientos tradicionalistas y religiosos, academias y sociedades de historia con disímil posicionamiento ideológico/político que, dentro de un espectro ideológico, iban desde la derecha hasta la izquierda. Estas diversas prácticas deben considerarse en relación con el contexto político e ideológico en el que se fueron formulando las corrientes revisionistas de la historia argentina (Galasso, 2004; Ramos, 2016). Podemos dividir este extenso periodo en varias etapas:

El lapso de tiempo que abarca esta etapa está caracterizado, en su mayor parte -de 1955 a 1973-, por el derrocamiento y la proscripción del

Primera etapa: De 1955 a 1976 peronismo y de su líder, Juan Domingo Perón, sucediéndose una serie de Gobiernos constitucionales y de facto. En 1973, el peronismo vuelve al poder hasta 1976. Sus partidarios contribuyeron en debates brindando apoyo a varias de las tendencias del Revisionismo histórico, tanto de derecha como de izquierda.

La producción de trabajos y obras generales que rebaten las versiones de la Historia oficial argentina durante estos años podría caracterizarse como un Revisionismo no oficial, que contaba con el apoyo de varios movimientos sociales. Esto se expresó en Vuelta de Obligado durante la década del cincuenta en la destrucción de monumentos por parte de fuerzas de la Marina de Guerra y, desde mediados de los años sesenta hasta mediados de los setenta, en la realización de conmemoraciones y actos 
cada 20 de noviembre, algunos de los cuales estuvieron signados por incidentes violentos entre diferentes facciones de izquierda y derecha.

Durante este espacio de tiempo las Fuerzas Armadas de la Argentina asaltaron el poder y lo ejercieron durante casi 8 años. El contexto general está dominado por los dictadores que se dividen en liberales y neo-liberales por un lado y nacionalistas de derecha por el otro. En Vuelta de Obligado, las conmemoraciones de la batalla fueron principalmente organizadas en el marco de actos oficiales de carácter militar.

En este lapso se desarrollan varios Gobiernos elegidos democráticamente; el contexto general que predomina es liberal y neo-liberal. Para inicios de la década de 1990 se impulsa por parte del Estado nacional la reivindicación oficial de la figura rosista mediante gestiones institucionales buscando promover una reivindicación "conciliadora" de su memoria (Shumway, 2010). En ese marco, en 1989 se impulsó la repatriación de los restos de Juan Manuel de Rosas - enterrado en Inglaterra-, evento que se festejó con actos oficiales y la reivindicación de la batalla de Vuelta de Obligado.

Espacio de tiempo en el que se generan nuevos movimientos revisionistas y otros se reciclan; se sancionan leyes respecto del patrimonio nacional y se ponen en relieve tanto figuras como hechos de las luchas por la emancipación de la Argentina. El contexto general es de debate entre los representantes de la historia oficial -generada durante

\section{Cuarta etapa: Desde mayo de 2003 hasta diciembre de 2015}

\section{Segunda etapa: De marzo de 1976 a diciembre de 1983}


los siglos XIX y XX- y las nuevas tendencias revisionistas que, en muchos casos, se manifiestan oficialmente y desde el poder político.

En el paraje de Vuelta de Obligado, se construyeron nuevos espacios recordatorios y estructuras alusivas al evento en los años 1979, mediados de 1980 (periodo de dictadura cívico-militar) y posteriormente, en 2008 y 2010.Un importante cambio en estas formas de memoria relacionadas con la batalla comenzó a darse desde finales del siglo XX debido a que, desde el Gobierno nacional, se tomaron importantes decisiones ideológicas de reconocimiento respecto de la batalla de la Vuelta de Obligado y la guerra del Paraná.

Se impulsó el desarrollo de instituciones oficiales de carácter revisionista y el reconocimiento de la Vuelta de Obligado como especial símbolo de la lucha anti-imperialista. De este modo se inició así un nuevo período de revisionismo histórico apoyado por el Estado en el marco del proceso del bicentenario de la independencia. A nivel provincial y municipal, se apoyó el proceso revisionista y de activación de la memoria de Obligado, incentivando su valor como recurso turístico y simbólico que posibilitaría potenciar el desarrollo local.

Como consecuencia de estos procesos de revisión se destacan una serie de eventos oficiales -y de amplia difusión- para activar la memoria de la batalla: en 1992 se sanciona la ley que declara a Vuelta de Obligado "Sitio Histórico Provincial"; en el año 2000 el Municipio 
de San Pedro promulga la ordenanza que establece la "zona de Reserva Natural y Refugio Histórico Natural"; en el 2002 la provincia lo declara "Reserva y Monumento Natural"; en el año 2008 se inauguró un museo de sitio en Vuelta de Obligado (en el que el equipo de Arqueología participó activamente colaborando para su formación); en el 2010 por decreto nacional se retomó la fecha de la batalla de Vuelta de Obligado para establecer un nuevo feriado nacional que conmemorara el día de la Soberanía nacional y, además, en el año 2010 se realizó un nuevo e imponente monumento inaugurado con presencia de la Presidenta de la Nación.

Todo lo anterior generó una mayor visibilidad del paraje de Vuelta de Obligado, ampliando el flujo turístico a la zona, y generando la consecuente oportunidad para algunos de poder desarrollar microemprendimientos locales. Además, tuvieron lugar algunas transformaciones de infraestructura que posibilitaron mejoras en la calidad de vida para los habitantes. Lo que nos interesa destacar de este proceso es que la memoria y la re-significación de este tipo de hechos históricos, en tanto conjunto de prácticas sociales que combinan la selección de recuerdos y olvidos, contribuyó a inscribir el presente de Vuelta de Obligado en una continuidad histórica -la historia nacional- reafirmada cada 20 de noviembre (Traverso, 2007). 
La dimensión pública de la investigación arqueológica en El Tonelero fue organizándose a partir de las relaciones del equipo de investigación con diferentes actores locales (representantes del Gobierno local, ONG ambientalista, vecinos del paraje, industrias locales). En todos los casos estas relaciones estuvieron mediadas por las representaciones sobre las implicancias de las investigaciones arqueológicas en Vuelta de Obligado, donde, como se explicó en el apartado anterior, el contexto revisionista de los últimos años tuvo importantes efectos materiales que permitieron abonar expectativas de crecimiento económico y mejora de la calidad de vida para los pobladores del paraje.

En este contexto, el trabajo arqueológico fue positivamente valorado porque, entre otras cosas, aportó elementos materiales para la memoria del acontecimiento, asociándola a un territorio específico. Estos aspectos nos recuerdan que en la actualidad cualquier forma de memoria histórica se encuentra ligada tanto con aspectos éticos y políticos como con procesos de mercantilización y espectacularización (Huyssen, 2000).

En concordancia, en el marco de los primeros acercamientos con las autoridades oficiales del lugar, a partir del año 2012, se mantuvieron conversaciones con la Directora del Museo Histórico de la ciudad de Ramallo, el Director de Desarrollo Social, el Intendente del municipio y varios concejales. Se tuvieron en cuenta las diferentes expectativas sobre los eventuales resultados de la futura investigación arqueológica; se

\section{La dimensión pública en la investigación arqueológica en el paraje El Tonelero}


propuso generar en El Tonelero una activación patrimonial en torno a la batalla similar a la de Vuelta de Obligado y con perspectivas respecto de impulsar el desarrollo local, enfatizando el valor económico y turístico del potencial sitio arqueológico. El encuentro con las autoridades locales se hizo público mediante los medios locales ( $\mathrm{TV}$, radio y prensa), estableciendo de esta manera un primer ámbito de interacción pública mediado por el Gobierno municipal.

No obstante, cuando llegamos a realizar las primeras tareas de prospección, nos encontramos con diferentes personas y vecinos del lugar que nos interpelaron con respecto a nuestro trabajo, mostrando desconfianza: ¿qué estábamos haciendo allí?; ¿a pedido de quién?; ¿con qué fin?, ¿éramos "agentes" del Gobierno municipal y tan corruptos como él? Aunque estas primeras consultas fueron resueltas de manera informal, a partir de entonces se sucedieron una serie de manifestaciones públicas en medios masivos (gráficos, audiovisuales), publicaciones digitales, redes sociales e intervenciones de los vecinos en el espacio público- que discutieron la presencia y los objetivos de la investigación arqueológica.

En este marco organizamos un encuentro y exposición en el Honorable Concejo Deliberante (HCD) de Ramallo con la participación de funcionarias del Instituto Cultural de la Provincia de Buenos Aires, para abrir el diálogo con los diferentes grupos involucrados e informar sobre los alcances y características del proyecto de investigación como comunicar 
aspectos técnicos y legales. Las discusiones generadas en y desde esa reunión nos permitieron reconocer la dimensión política de nuestro trabajo arqueológico: la propuesta de estudio en el sitio El Tonelero se había convertido en un asunto de discusión pública y política que se articulaba con disputas previas respecto al uso del espacio en que se anclaba la investigación. Las diferentes voces que se hicieron visibles marcaron una confrontación con respecto a ¿cómo definir y utilizar el espacio que estaría vinculado con el campo de batalla? y ¿de qué manera abordar la conmemoración del acontecimiento?

Actualmente estas discusiones se organizan en dos posiciones principales. Por un lado, actores que reivindican la lucha por la defensa del territorio con el fin de vivir y promover políticas de conservación de la historia local y su medio ambiente -demandas que también se habían registrado en Vuelta de Obligado- y, por el otro, actores que buscan acotar el espacio de memoria para habilitar otro tipo de usos en el área asociada a la investigación -principalmente la explotación portuaria e industrial(Figura 1). 


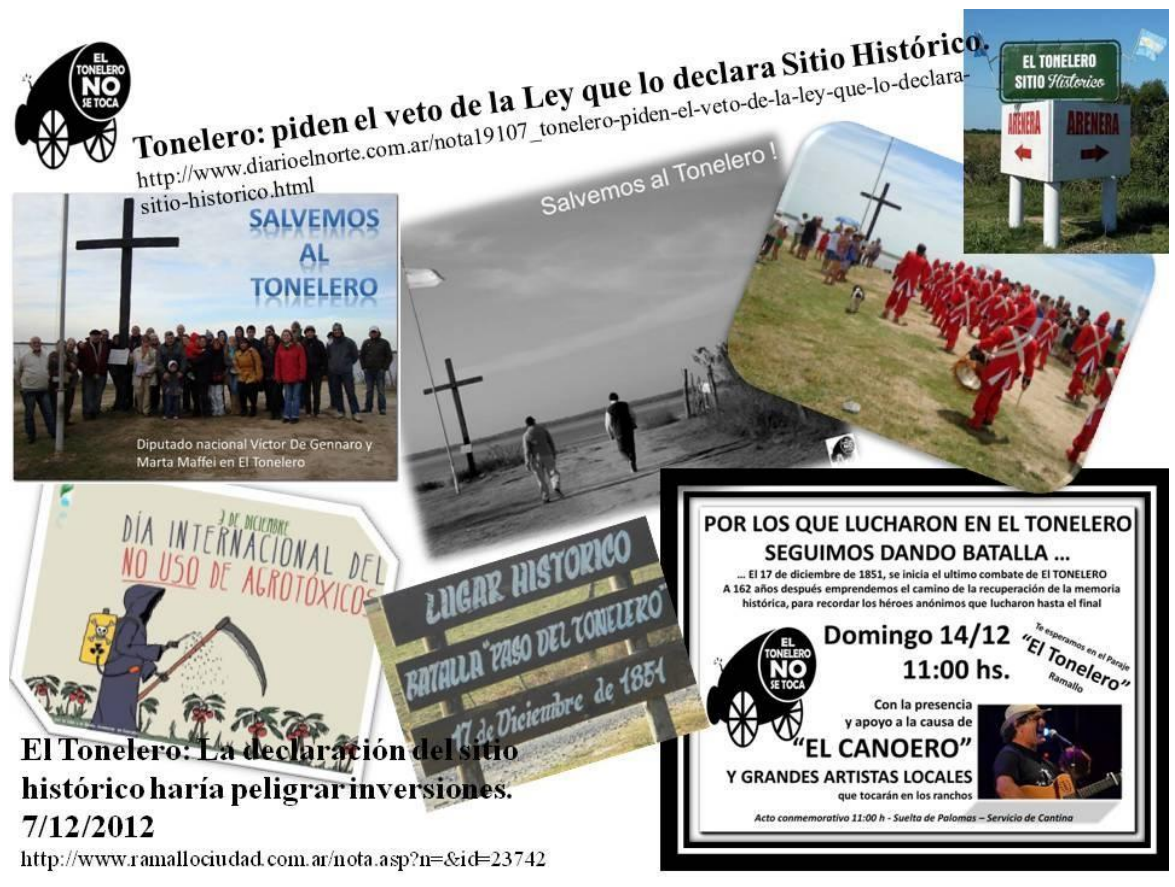

Figura 1. Expresiones públicas en torno al paraje El Tonelero, lugar donde se inició la investigación arqueológica.

Fuente: Elaboración propia.

En estos movimientos, el pasado fue abordado en función de hacer posible un determinado presente. A su vez, la conmemoración constituyó una herramienta que, por un lado, sirvió para ratificar y generar elementos legitimadores y cohesionantes pero paralelamente, también contribuyó a dividir y confrontar a los vecinos entre sí, ya que muchos no están de acuerdo con alentar algún tipo de interpretación del pasado. Toda esta 
situación mencionada, sin duda tuvo fuertes repercusiones en los conflictos actuales sobre el uso del espacio en torno al paraje como, por ejemplo, la decisión de inaugurar o no un monumento que recuerde los hechos históricos ocurridos. Este tipo de interpretaciones deben ser entendidas como parte de la disputa simbólica en la cual los sujetos obtienen su reconocimiento jurídico y social y su entrada en la tradición.

En este caso, las disputas se organizan por un lado considerando la posibilidad de que El Tonelero forme parte de la conmemoración que en la actualidad se ancla en Vuelta de Obligado y, por el otro, entre vecinos del paraje en función de diferentes expectativas sobre el uso del territorio. En relación con ello, Andrea Huyssen ha observado una estrecha relación entre memorias locales y las luchas que buscan "nutrir y expandir el espacio habitable en lugar de destruirlo en aras de alguna promesa futura" (Huyssen, 2000, p. 19).

En este proceso, el pasado se recupera como conjunto cerrado cuyo valor reside más en la posibilidad de conmemoración que en su contenido histórico. De esto resulta un discurso ambiguo del pasado reivindicado que confunde acontecimientos, de hecho en El Tonelero observamos que las disputas públicas hacen referencia a tres batallas del siglo XIX: dos batallas ocurridas en el marco de la guerra del Paraná, el 9 de enero y el 10 de febrero de 1846; y una batalla de la guerra con el Brasil que tuvo lugar el 17 de diciembre del año 1851. 
Es llamativo y ejemplificador el hecho de que el acontecimiento más revalorizado por los locales es la guerra de Paraná como estandarte de la lucha por la soberanía nacional, pero el día elegido para realizar los actos conmemorativos es el 17 de diciembre de cada año, fecha que no está asociada con la reivindicación de aquellos intereses aunque es una fecha de defensa que hacen los federales de Rosas frente a otra incursión extranjera. De este modo, se observa cómo en la selección del pasado operan mecanismos que exceden los límites y divisiones impuestas por la historia oficial y, por otra parte, cómo la categoría del recuerdo no está interpelada por los cánones académicos.

En este contexto la investigación arqueológica fue adquiriendo diferentes sentidos en el espacio local, de acuerdo con el modo en que se desenvolvió el conflicto en torno al uso del espacio en El Tonelero. Inicialmente, el escaso conocimiento público sobre los objetivos y naturaleza del proyecto de investigación arqueológica fue objeto de discusión, principalmente por los vecinos del paraje. Luego de la reunión pública y de que las características del proyecto de investigación fueran conocidas por los diferentes actores involucrados en las disputas, el lugar de la investigación arqueológica se modificó: todos los actores, por lo menos desde el discurso, estuvieron de acuerdo con que se realice la investigación arqueológica y expresaron una fuerte y urgente demanda por establecer los límites precisos del sitio arqueológico. 
Esta identificación es planteada en términos de señalamiento, de marca territorial cuyo potencial reside en constituir un basamento para la identificación de la historia del lugar con la guerra del Paraná y las batallas en defensa del territorio. De este modo, la demarcación de los límites de los sucesos históricos actúa como verdad legitimadora frente a los posibles usos del paisaje, frente a esto, la memoria y la selección de los eventos a reivindicar juegan un papel central en la construcción de narrativas presentes en torno al pasado. Ejemplo de las posibles situaciones que se pueden producir a partir de estas tensiones, son los hechos que ocurrieron en el paraje hacia fines del 2014, cuando los vecinos construyeron una cruz de hierro y madera que emplazaronjusto en el lugar que se pretende construir el puerto. Junto a esta colocaron una placa conmemorativa a los caídos en el combate por la lucha por la soberanía nacional.

Un año más tarde, la Municipalidad erige un monumento conmemorativo al Gral. Lucio Mansilla, quien fue el encargado de liderar las tropas de la Confederación, a dos kilómetros aproximadamente alejado del punto señalado por los vecinos y los ambientalistas para situar la cruz. El objetivo, detrás de esta acción, fue legitimar como lugar de memoria del evento a otro sector del paisaje, que no presentaba ningún interés para concretar el puerto.

En este proceso, la Arqueología es posicionada en el lugar de la voz científica legítima que permitiría establecer un criterio "neutral” para 
dirimir el conflicto. El espacio potencialmente vinculado con la investigación histórica es reclamado por los diferentes actores para ser comprendido en términos de patrimonio histórico. La identificación del espacio en disputa como patrimonio histórico conllevaría la suspensión de intereses particulares en pos de un supuesto interés general de carácter común: su cuidado, conservación y su disfrute. A su vez, este proceso implicaría establecer normas de uso y acceso, así como la intervención de especialistas que las gestionarían. Sobre la base de estos argumentos, los conflictos se desenvuelven en torno a las iniciativas para la declaración de sitio histórico del paisaje de El Tonelero adyacente al río, lo que se había logrado a través de una Ley de la Provincia de Buenos Aires, que luego fue vetada mediante gestiones del Gobierno municipal con el Gobierno provincial.

Frente a esta situación, los resultados de las investigaciones arqueológicas son exigidos como si de ello dependiera la posibilidad de establecer los límites territoriales para organizar la conmemoración proyectada. Nos preguntamos en qué medida estas relaciones y demandas que los actores locales establecieron frente al proceso de investigación arqueológica pueden pensarse como prácticas de la memoria, pues otorgan a la actividad arqueológica en El Tonelero significados específicos que conectan el acontecimiento histórico investigado con las necesidades actuales de los habitantes del paraje. 
En el caso presentado, el equipo de Arqueología fue interpelado casi a la manera de un juez, asumiendo que el establecimiento de límites en torno al sitio arqueológico permitiría dirimir las discusiones, que como detallamos anteriormente, se están desarrollando en el plano jurídico, político y social. Esto es un problema para nosotros debido a que, entre otras cuestiones, establecer dichos límites es uno de los principales inconvenientes que se plantea en el estudio arqueológico de los campos de batalla de época moderna: debido a la complejidad de las estrategias bélicas, las tecnologías puestas en juego, su distribución en el espacio y los procesos de formación que actúan en los sitios arqueológicos, muchos de los cuales, como es el de nuestro caso de estudio, insertos actualmente en contextos rururbanos que a través del tiempo codificaron considerablemente el paisaje.

Incluso en el caso de que los límites pudieran ser rápida y fácilmente identificables, es posible discutir la validez de estos datos para el establecimiento de criterios de verdad y justicia que posibiliten dirimir el conflicto sobre el uso del espacio. Esta situación nos llevó a considerar la relación entre investigación histórica y establecimiento de verdad y justicia, tema que ha sido ampliamente trabajado desde los estudios de la memoria (Traverso, 2007; Ginzburg, 1993; Foucault, 1980; entre otros).

La verdad histórica y la verdad jurídica son muy diferentes; la verdad del historiador no es solamente provisoria y precaria, sino que es también más

\section{Conclusiones: Política y quehacer arqueológico}


problemática. Resultado de una operación intelectual, la Historia es analítica y reflexiva, trata de mostrar las estructuras subyacentes a los acontecimientos, las relaciones sociales en las cuales están implicados los seres humanos y las motivaciones de sus actos (Ginzburg, 1993). En la medida en que pudimos explicitar estas diferencias y aclarar que los límites del campo de batalla no podían establecerse de forma inmediata ni definitiva, nuestro rol en la discusión fue variando y pasamos a conformar un actor más dentro de la disputa.

Entonces, pudimos actuar a partir de nuestro reconocimiento como sujetos políticos, generando un desplazamiento del lugar de neutralidad socialmente impuesta como parte de la herencia moderna en torno a las representaciones de la ciencia, a un posicionamiento en relación con nuestros intereses: investigar y que no se destruya el potencial sitio arqueológico, acompañar la lucha de los vecinos y distanciarnos de las perspectivas del Gobierno local que promueven el desarrollo de proyectos industriales invasivos -lo que para el equipo de Arqueología implicaría una intervención fuerte en un espacio en el que hay potencialmente un sitio arqueológico-.

De este modo, las investigaciones arqueológicas en El Tonelero avanzaron con el apoyo de parte de los vecinos y las organizaciones ambientalistas, quienes acompanaron nuestra presencia en el campo para el desarrollo de los trabajos de prospección durante fines de 2012 y durante todo el año 
2013 (Helfer y Raies, 2012; Ramos et ál., 2013; Raies y Ramos, 20142015). Estos trabajos incluyeron la realización de sondeos exploratorios, transectas de recolección de superficie y transectas con detector de metales. La mayoría de los objetos hallados corresponden a materiales del siglo XIX posiblemente vinculados a contextos domésticos; en menor proporción se hallaron restos de ocupaciones indígenas y materiales que podrían corresponder con los conflictos bélicos desarrollados en la zona a mediados del siglo XIX, en los años 1846 y 1851, respectivamente, tales como metralla, vidrio y clavos de sección cuadrangular; asimismo, pueden dar cuenta de la ocupación en forma continua, la cual se desarrolló entre los años de 1838 y 1846, del Campamento Estable de la División del Norte. Como se puede observar a partir de lo expuesto, se trata de un palimpsesto de difícil resolución debido a que en los eventos bélicos que tuvieron lugar en El Tonelero, se usaron armamento y objetos de uso cotidiano relativamente similares.

Por su parte, los restos materiales indígenas corresponden a una ocupación previa, probablemente por espacio de un período prolongado aunque todavía no bien determinado (Ramos et ál., 2013). El avance de los trabajos de investigación se demoró por las lentas gestiones del Gobierno local, frente a esto, el equipo de Arqueología impulsó una serie de reclamos, junto con parte de los vecinos y ambientalistas, para que se realicen controles de impacto integral en las zonas donde se 
proyectan realizar emprendimientos industriales que hasta la fecha también han sido demorados.

Por estos motivos, las investigaciones arqueológicas en torno al sitio El Tonelero continúan en una fase inicial en la que adquiere cada vez mayor relevancia la evaluación de los métodos de prospección y sondeos que posibilitarán realizar una aproximación del lugar donde pudieron desarrollarse los enfrentamientos y/o de aquellos lugares claves como el campamento y el hospital de campaña (Ramos et ál., 2014).

Recientemente -1 de septiembre de 2016- se ha vuelto a tomar contacto con las nuevas autoridades políticas municipales, en particular con el Intendente (que fue concejal hasta diciembre de 2015) y el Presidente del HCD de Ramallo. Ambos solicitaron la opinión del director del proyecto general de investigación sobre la guerra del Paraná acerca de la instalación de obras -puerto, empresas, etc.- en el lugar. Vale aclarar que la gestión política anterior del municipio estableció y firmó acuerdos precontractuales, con algunas empresas, para construir instalaciones en el área que aún no se han realizado.

En una reunión realizada en el despacho del jefe comunal, el interpelado nuevamente aconsejó a las autoridades el realizar un estudio de impacto integral, tal como se había realizado en el sitio Vuelta de Obligado en 2010, que abarque no solamente la instancia arqueológica sino que también considere el estudio que pueden llevar a cabo profesionales de otras 
disciplinas científicas, debido a que no es solamente un determinado y particular registro arqueológico el que puede ser afectado por las grandes obras, sino todo el ambiente.

Para finalizar, entendemos que en este caso la discusión se organiza a partir de lo que Traverso (2007) llama "usos público de la memoria", pues, al igual que el historiador, como arqueólogos contribuimos a formar y orientar la memoria. En este sentido, el proceso de patrimonialización de la memoria implica la imposición de unos significados por sobre otros. En nuestro caso, como arqueólogos profesionales, portadores de un saber específico, somos interpelados por distintos actores con intereses disímiles en busca de una pretendida "verdad" (definición de sitio arqueológico) que justifique y sirva de plataforma para acciones posteriores.

Si a esto le agregamos que el hallazgo de un determinado sitio justificaría su reconocimiento jurídico como sitio histórico y de esta manera una porción del paisaje pasaría a pertenecer al patrimonio de la nación, posibilitando su uso/aprovechamiento como "bien público", entonces nos preguntamos si como arqueólogos ¿ayudamos a la construcción de una memoria no monolítica, plural e inevitablemente conflictiva? Consideramos como hemos señalado arriba, que solo puede buscarse una respuesta al posicionarnos como sujetos políticos en un determinado contexto, en donde la dimensión pública de la investigación arqueológica posibilite espacios de discusión, diálogo y resolución. 
Por último, destacamos que la situación de El Tonelero no es un caso aislado. En nuestras investigaciones también hemos experimentado situaciones relativamente similares en Vuelta de Obligado. Fuera de la región pampeana tenemos los casos de Norpatagonia, como los sitios del Río Limay afectados por las grandes obras hidroeléctricas, otras de infraestructura y las actividades económicas que incidieron sobre los pobladores y los sitios arqueológicos. En este último caso, a las modificaciones estructurales se sumaron el coleccionismo y el saqueo que contribuyeron a su destrucción (Ramos, 2013).

Al igual que en El Tonelero, en diferentes lugares de Latinoamérica se vienen documentando cada vez con mayor frecuencia, situaciones complejas en donde diferentes grupos de la sociedad civil desafian la práctica arqueológica demandando no solo a los investigadores compromisos sociales y políticos sino también requiriendo la intervención de las instituciones a cargo de la gestión patrimonial.

Pueden consultarse por ejemplo, Asensio (2013) sobre la situación en Perú y la emergencia de diferentes modelos vinculados con la participación comunitaria para la gestión del patrimonio arqueológico. En el litoral uruguayo, Brum Bulanti (2013) analiza los procesos de negociación entre diferentes colectivos de la sociedad civil, arqueólogos y representantes del Gobierno frente a la gestión del patrimonio en el marco de una investigación arqueológica; en Coatlichan, México, Rozental (2011) 
documentó intensos procesos de apropiación y re-significación social en torno al Monolito de Tláloc que dan cuenta de los procesos de negociación y conflictos actuales de los que forman parte los objetos arqueológicos. En muchos lugares de nuestro continente la propiedad de la tierra y la gestión de sus recursos conforman la plataforma de la relación conflictiva entre los diferentes actores de la comunidad, arqueólogos y gestores del patrimonio arqueológico (Ayala Rocabado, 2005; Lima Tórrez, 2003; Londoño, 2016; Woynar, 2003; entre otros).

Acordamos con Menezes Ferreira (2015) en que estos contextos ponen de manifiesto la necesidad de dirigir el quehacer profesional hacia una gestión compartida de "las cosas arqueológicas", visibilizando una serie de contradicciones en las que nuestro quehacer profesional se encuentra inmerso. No solo porque las legislaciones arqueológicas establecen límites fijos para la gestión e interpretación de los objetos que excluyen a diferentes colectivos sociales implicados con los mismos, sino porque una práctica de gestión compartida requiere repensar los parámetros temporales, metodológicos y epistemológicos a partir de los cuales se desarrolla la investigación arqueológica.

En suma, se trata de escenarios complejos en los que el diálogo es de vital importancia para una mejora de las relaciones, en tanto promueve un cambio en la forma de interacción y en la manera en que algunos actores consideran el quehacer arqueológico. En nuestra experiencia, el desarrollo 
de un trabajo dialógico e interactivo con los mismos sujetos en distintas instancias, junto con la toma de posición de nuestro equipo, posibilitó poder ver la heterogeneidad de posturas existentes dentro del espacio local y, a partir de esto, "desesencializar" tanto a las comunidades como a los investigadores -en el sentido de dejar de entenderlos como grupos homogéneos-. Creemos que este tipo de diálogos y encuentros con los actores locales son esenciales para generar relaciones de cooperación que apunten a contribuir con conocimiento y prácticas, a la consecución de objetivos que sean relevantes para el desarrollo local. 
Asensio, R. H. (2013). ¿De qué hablamos cuando hablamos de participación comunitaria en la gestión del patrimonio arqueológico? Revista Argumentos, 3. Recuperado de http://revistaargumentos.iep.org.pe/articulos/de-que-hablamoscuando-hablamos-de-participacion-comunitaria-en-la-gestiondel-patrimonio-cultural/

Astorga, M. (2007). Patrimonio y comunidad: el caso de Vuelta de Obligado. En E. Néspolo, M. Ramos M. y Goldwaser B. (Eds.), Signos en el tiempo y rastros en la tierra.V Jornadas de Arqueología e Historia de las regiones pampeana y patagónicas, Vol. 2 (pp. 120-124). Luján Universidad Nacional de Luján, Departamento de Ciencias Sociales.

Ayala-Rocabado, P. (2005). Pueblos originarios y arqueología: discursos en torno al patrimonio arqueológico en San Pedro de Atacama (Segunda Región de Chile). Textos Antropológicos, 15(2), 249-261.

Brum-Bulanti, L. (2013). Gestión del patrimonio arqueológico en el litoral oeste del departamento de Maldonado (Uruguay). La investigación como práctica integral. Revista del Museo de La Plata, 13(87), 417-428. Recuperado de http://www.fcnym.unlp.edu.ar/uploads/docs/rmlp_antro_2013_t 13_n87_brun.pdf 
Cattaruzza, A. (2007). Los usos del pasado. La historia y la política argentinas en discusión, 1910-1945. Buenos Aires: Editorial Sudamericana.

Devoto, F. y Pagano, N. (2009). Historia de la Historiografía Argentina. Buenos Aires: Sudamericana.

Ermaccora, J. y Revello, C. A. (2009). Nota de los editores: La vuelta del Restaurador, 30 de septiembre de 1989. Folios Numismáticos, 39, 4-7. Recuperado de http://www.numisma.es/CENUSA/B39Septiembre2009.pdf

Foucault, M. (1980 [1973]). La verdad y las formas jurídicas. Barcelona: Gedisa.

Galasso, N. (2004). De la Historia Oficial al Revisionismo Rosista. Corrientes historiográficas en la Argentina. Cuadernos para otra historia. Buenos Aires: Centro Cultural "Enrique Santos Discépolo".

Ginzburg, C. (1993). El juez y el historiador. Consideraciones al margen del proceso de Sofri. España, ANAYA S.A.

Helfer, V. y Raies, A. (2012). Primer Informe. El Tonelero, Ramallo, Provincia de Buenos Aires. Universidad Nacional de Luján. 
Huyssen, A. (2000). Medios, política y memoria. Revista Puentes, 2, 12-29. Recuperado de http://www.memoriaenelmercosur.educ.ar/wpcontent/uploads/2009/04/puentes02.pdf

Iazetta, O. (2008). Lo público, lo estatal y la democracia. Revista Iconos, 32, 49-60. $\quad$ Recuperado de http://www.redalyc.org/articulo.oa?id=50903206

Jacubowicz, E. (2005). Las visiones del pasado o la historia argentina a través del cine 1933-2003. Algunos comentarios y reflexiones. En Actas X Jornadas Interescuelas, Departamentos de Historia, (s/n). Universidad Nacional de Rosario: Publicación digital.

Lima-Tórrez, M. del P. (2003). Participación comunitaria, desarrollo sostenible y arqueología: el caso de Quila Quila (Chuquisaca, Bolivia). Chungara, Revista de Antropología Chilena, 35(2), 361-365. Recuperado de: http://www.scielo.cl/scielo.php?script=sci_arttext\&pid=S071773562003000200017

Londoño, W. (2016). Arqueología por contrato y nuevos contratos arqueológicos. Revista Jangwa Pana, 15(1), 117-128. Recuperado de http://revistas.unimagdalena.edu.co/index.php/jangwapana/articl e/view/1756 
Menezes-Ferreira, L. (2015). Las cosas están vivas: relaciones entre cultura material, comunidades y legislación arqueológica. Complutum, 26(1), 37-45.

Moore, B. (1984). Privacy: studies in social cultural history. Armonk: Nueva York ME Sharp Ink.

Ortiz, R. (2002). Globalización y esfera pública. Entre lo nacional y lo transnacional. Revista Signo y Pensamiento, 21(41), 68-81. Recuperado de http://www.redalyc.org/articulo.oa?id=86011596008

Rabotnikof, N. (1996). Memoria y política: Compromiso ético y pluralismo de interpretaciones. Revista Uruguaya de Ciencia Política, 9, 143-150. Recuperado de http://cienciassociales.edu.uy/wpcontent/uploads/sites/4/2013/archivos/20071109222946.pdf

Ramos, M. (2009). Otras miradas de la realidad. Para ir más allá de la materialidad del pasado. En Actas Coloquio binacional argentino-peruano. Perspectiva latinoamericana (pp. 41-70). Buenos Aires: Instituto Superior del Profesorado Dr. Joaquín V. González, Centro de Investigaciones Precolombinas.

Ramos, M. (2013). Patrimonio arqueológico de la cuenca del Río Limay. Agentes y procesos que lo destruyen. Atek-Na [En la tierra], 3,75-110. 
Ramos, M. (2016). Huellas en la arena. Conocimientos, contextos y algo más. Luján: Universidad Nacional de Luján.

Ramos, M., Helfer, V., Lanza, M., Bognanni, F., Raies, A., Hernández de Lara, O., Alanís, S., Pinochet, H. y Umaño, M. (2013). Arqueología histórica de la guerra del Paraná. Cuadernos de Antropología, 10, 403-423.

Ramos, M., Lanza M., Helfer, V., Bognanni F., Raies A., Darigo M., Dottori C., Warr M., Santo, C., Raño, J., Hernández de Lara, O., Pinochet, H., Alanís, S. y Umaño, M. (2014). Arqueología histórica de la Guerra del Paraná: la de Vuelta de Obligado y el Tonelero. En C. Landa y O. Hernández de Lara (Eds.), Sobre campos de batalla. Arqueología de conflictos bélicos en América Latina (pp. 75-108). Buenos Aires, Aspha ediciones.

Ramos, M. y Raies, A. (2013). Segundo Informe. Proyecto de investigación. Informe sobre el sitio arqueológico de El Tonelero, Ramallo, Provincia de Buenos Aires.

Raies, A. y Ramos, M. (2014-2015). Tercer Informe sobre el sitio arqueológico de El Tonelero, Ramallo, Provincia de Buenos Aires.

Rozental, S. (2011). La creación del patrimonio en Coatlinchan: Ausencia de piedra, presencia de Tláloc. En P. EscalanteGonzálbo (Coord.), La idea de nuestro patrimonio histórico y 
cultural (pp. 341-361). México: Dirección General de publicaciones del Consejo Nacional para la Cultura y las Artes.

Salerno, V. (2014). Trabajo arqueológico y representaciones del pasado prehispánico en provincia de Buenos Aires. Buenos Aires: Editorial de Publicaciones de la Facultad de Filosofía y Letras, Universidad de Buenos Aires.

Salerno V. y González M. I. (2014). Conocimiento en relación. Reflexiones sobre el trabajo de campo arqueológico en el curso medio e inferior del río salado bonaerense. Revista del Museo de Antropología Universidad Nacional de Córdoba, 7(1), 25-38. Recuperado de https://revistas.unc.edu.ar/index.php/antropologia/article/viewFil e/9092/10189

Shumway, J. (2010). “A veces saber olvidar es también tener memoria”: La repatriación de Juan Manuel de Rosas, el menemismo, y las heridas de la memoria Argentina. En O. Barreneche y A. Bisso (Comps.), El tiempo pasa, la historia queda. Ayer, hoy y mañana son contemporáneos (pp. 93-132). Buenos Aires: Editorial de la Universidad Nacional de La Plata. Recuperado de http://historiapolitica.com/datos/biblioteca/muerte\%20y\%20poli tica_shumway.pdf 
Traverso, E. (2007). Historia y Memoria, notas sobre un debate. En F.

Levin y M. Franco (Eds.), Historia Reciente: perspectivas y desafios para un campo en construcción (pp. 67-96). Buenos Aires: Paidós.

Woynar, M. (2003). Arqueología y problemática social: Hacia un manejo de los recursos arqueológicos con mayor colaboración de las comunidades. En J. P. Laporte, B. Arroyo, H. Escobedo y H. Mejía (Eds.), XVI Simposio de Investigaciones Arqueológicas en Guatemala, 2002 (pp. 36-47). Guatemala: Museo Nacional de Arqueología y Etnología. Recuperado de: http://asociaciontikal.com/pdf/06.02\%20-

\%20Marion\%20Woynar\%20-\%20en\%20PDF.pdf

\section{¿Cómo citar este artículo?}

Salerno, V., Umaño, M., Helfer, V., Raies, A., Pinochet, H. C., Darigo, M., Warr, M. y Ramos, M. (Enero-junio, 2017). Cuando los límites importan: Dimensión pública e implicancias sociales del trabajo arqueológico. Revista humanidades, 7(1), 1-37. doi: http://dx.doi.org/10.15517/h.v7i1.27640 